EPJ Web of Conferences 53, 04003 (2013)

DOI: $10.1051 /$ epjconf/20135304003

(C) Owned by the authors, published by EDP Sciences, 2013

\title{
The Yakutsk array experiment: Main results and future directions
}

\author{
Anatoly Ivanov ${ }^{\mathrm{a}}$ for the Yakutsk array group ${ }^{\mathrm{b}}$ \\ Shafer Institute for Cosmophysical Research \& Aeronomy, Lenin Avenue, 31, Yakutsk \\ 677980, Russia
}

\begin{abstract}
The results of the Yakutsk array experiment aimed at investigations of ultra-high energy cosmic rays (UHECRs) are presented. We outline the current status of the instrument and an outlook for future directions and areas of study.
\end{abstract}

\section{INTRODUCTION}

- In 1959 D.D. Krasilnikov has formulated an idea of the EAS array in Yakutsk [1];

- 1967: The construction begun of EAS-13 prototype;

- 1970: Engineering stage built up; first showers detected;

- To 1973 the $1^{\text {st }}$ stage array was completed consisting of 43 stations with 2 scintillators, Cherenkov light detectors, detectors of muons;

- 1991: the $2^{\text {nd }}$ stage of the array completed. Infill array of 18 stations, and a large area muon detector were added;

- In 2005 the array modernization program was approved.

\section{PRESENT STATUS OF THE YAKUTSK ARRAY EXPERIMENT}

A schematic view of the present layout of the array detectors and photos of the main detectors are shown in Figs. 1, 2. 58 stations are distributed within the triangular hierarchical grid of the total area ${ }^{1}$ $10 \mathrm{~km}^{2}$. The three main components of EAS are detected with scintillators, 6 muon detectors and 48 air Cherenkov light detectors $[2,3]$. The shower events are selected by the coincidence of signals from $n \geq 3$ stations, which in turn have triggered by the two scintillation detectors in each station.

Actually, all detectors of the array are obsolete, energy estimation and angular resolution accuracies are insufficient; the array is urgently needed for modernization [4].

\section{MAIN RESULTS AND FUTURE DIRECTIONS OF INVESTIGATIONS}

In this section we briefly overview some results of the Yakutsk array experiment concerning, mainly, astrophysics of UHECRs and our purposes for a future.

\footnotetext{
ae-mail: ivanov@ikfia.ysn.ru

${ }^{b}$ For the full authorlist see Appendix "Collaborations" in this volume.

$18.2 \mathrm{~km}^{2}$ since 2010
}

This is an Open Access article distributed under the terms of the Creative Commons Attribution License 2.0, which permits unrestricted use, distribution, and reproduction in any medium, provided the original work is properly cited. 


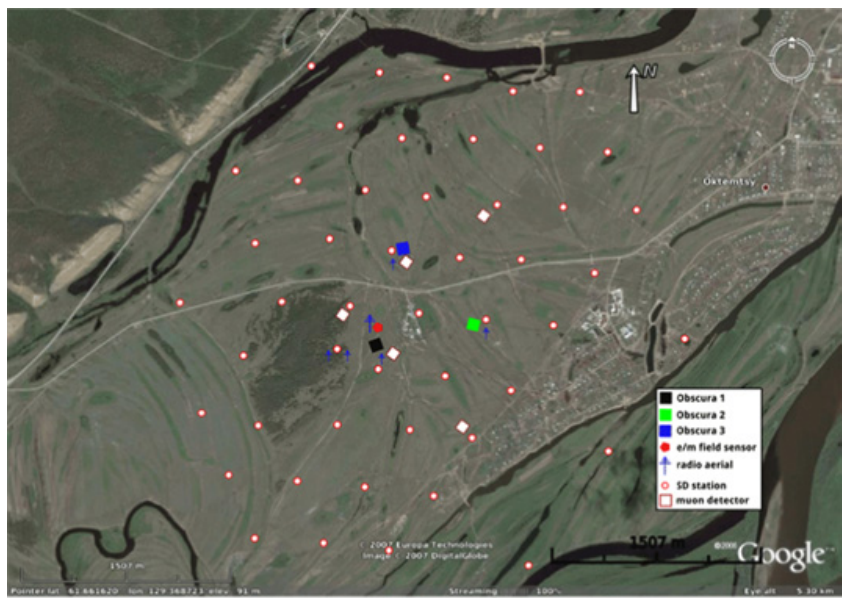

Figure 1. The map of the Yakutsk array.

Energy spectrum of CRs measured with the Yakutsk array in the energy range $E \in\left(10^{15}, 10^{20}\right) \mathrm{eV}$ is shown in Fig. 3 in comparison with some other relevant experiments. All the data are in agreement with the 'knee', 'ankle' and GZK suppression features in the spectrum [5], if to correct CR intensities observed and to scale the energies estimated by the arrays, for instance, along suggestion [6].

The mean shower maximum depth in the atmosphere, $X_{\max }$, is estimated using the connection between this parameter and the lateral distribution slope of Cherenkov light intensity and the halfwidth of the signal at the ground level [7] (Fig. 4). This data are used to estimate the average mass of CR particles using Horandel's relation [8] (Fig. 5). The $X_{\max }$ positions are calculated with QGSJet II-03 and SIBYLL 2.1 models, while the expected GCR composition is given by the SNR model [9]. A comparison with GCR simulation results demonstrates a consistent behavior of $\langle\ln A\rangle$ changing with energy: in the range $E \in\left(10^{15}, 10^{16}\right) \mathrm{eV}$ the composition becomes heavier, the average mass gets its maximum, then the composition becomes lighter above the energy $\sim 3 \times 10^{16} \mathrm{eV}$.

Arrival directions of UHECRs detected with the Yakutsk array were analyzed in conjunction with the data from other arrays searching for correlation with the two large-scale structures in the nearby universe - Milky Way disk within the Galaxy and the supergalactic plane (SGP) in the local galactic field [10]. We have used the (super)galactic plane enhancement parameter, $R$, defined as

$$
R=\frac{n(|b|<d)-n(|b| \geq d)}{n(|b|<d)+n(|b| \geq d)}, \delta R=2 \sqrt{\frac{n(|b|<d) n(|b| \geq d)}{(n(|b|<d)+n(|b| \geq d))^{3}}},
$$

where $b$ is the (super)galactic latitude; $d=10^{0}$ is the plane border. The results are shown in Fig. 6 taken from [11], in comparison with an expected-for-isotropy $R_{0}$ calculated by the Monte Carlo method $\left(N=10^{6}\right)$. There is no statistically significant excess flux from the galactic disk and SGP in the whole energy range, but it seems to be a systematic increase of the UHECR flux from the SGP according to the Yakutsk array data which is supported by the data from AGASA and PAO above $4 \times 10^{18} \mathrm{eV}$ : all the observed enhancement parameters are greater than those expected in the isotropic case.

Distribution of $X_{\max }$, namely, the slope of distribution, $\Lambda$, beyond the peak (Fig. 7, upper panel), can be used to estimate the total inelastic proton-air cross-section, $\sigma_{p-a i r}$, using model simulation of the relation $\Lambda=K \sigma_{p-a i r}$ [12]. Basing on our measured $X_{\max }$ distributions (Fig. 7, bottom panel) at energies $3.3 \times 10^{15} \mathrm{eV}, 7.2 \times 10^{15} \mathrm{eV}, 2.1 \times 10^{16} \mathrm{eV}$, we have estimated proton-air cross-section [13]. Figure 8 shows a compilation of the $\sigma_{p-a i r}$ data obtained in different experiments together with model simulation 


\section{UHECR 2012}
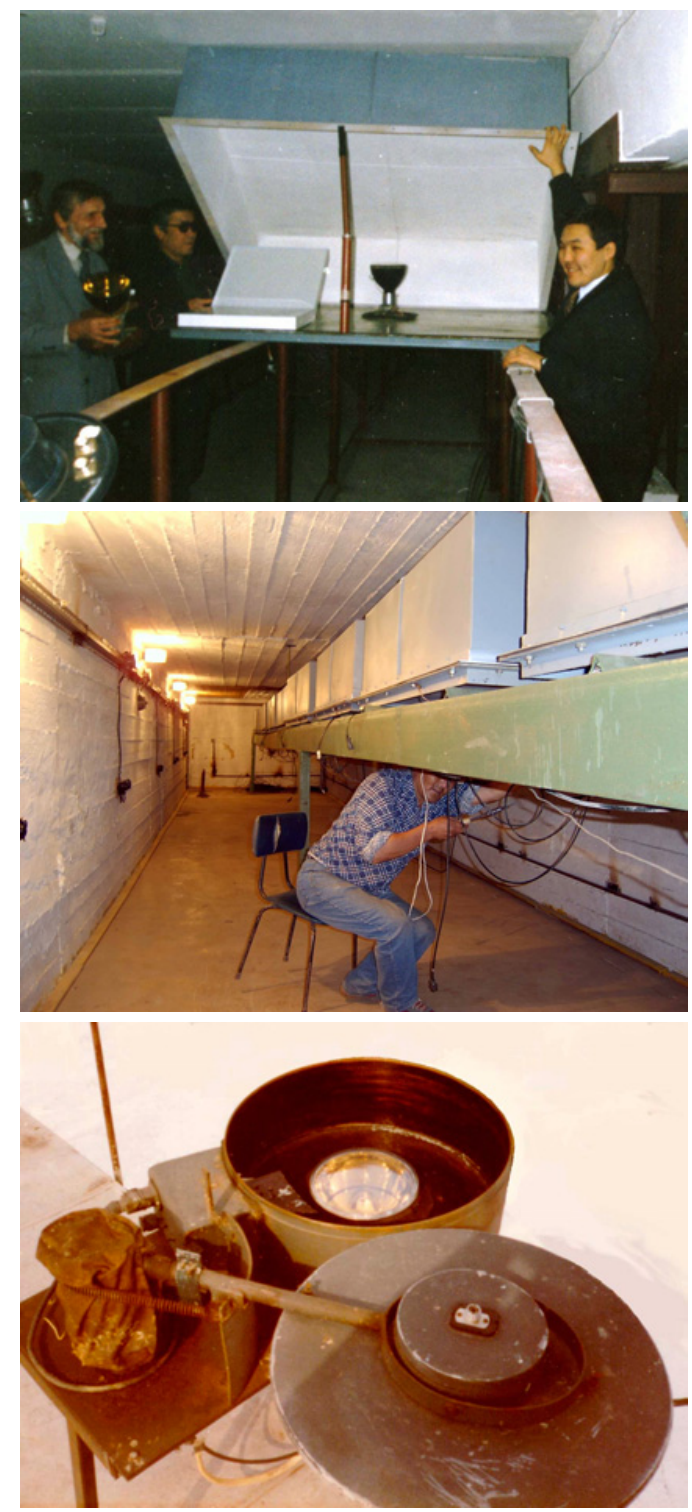

Figure 2. Main detectors of the array: scintillator (top); muonic (middle); Cherenkov (bottom).

results in the energy range $\left(10^{11}, 10^{20}\right) \mathrm{eV}$. Our previous estimations [14] are shown, as well. As it is seen from the Figure, CR data together with accelerator data set some limits on hadronic interaction models used to describe EAS development at the highest energies.

Our plans for a future: we are going to radically modernize the array in order to have an instrument precise enough for measuring the highest energy galactic (G) CRs - their sources, energy spectrum, and mass composition (Fig. 9). A related aim is to study a transition region between $\mathrm{G}$ and extragalactic (EG) components of CRs where some irregularities in spectrum and composition may be revealed. At the same time, we are going to join efforts with CR community in construction of a next-generation ground based giant detector of EG CRs. 

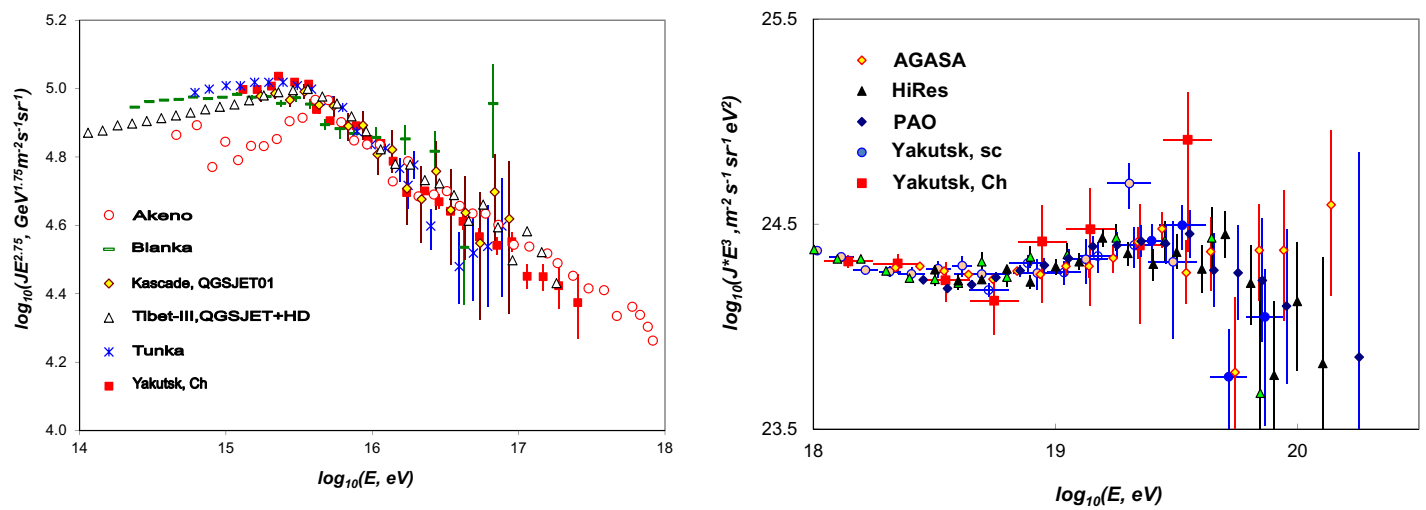

Figure 3. Differential energy spectrum of CRs below (left panel) and above (right panel) $E=10^{18} \mathrm{eV}$ measured with the Yakutsk array are shown in comparison with other experiments. CR intensity and energy are corrected for systematic errors. Details are given in the text.

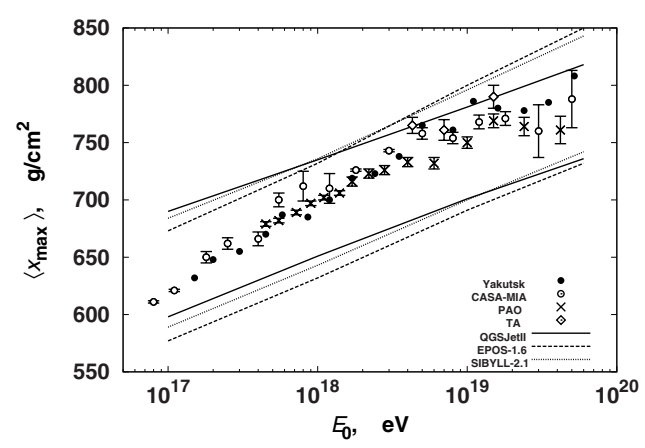

Figure 4. Depth of EAS maximum: measurements and models at $E>10^{17} \mathrm{eV}$.

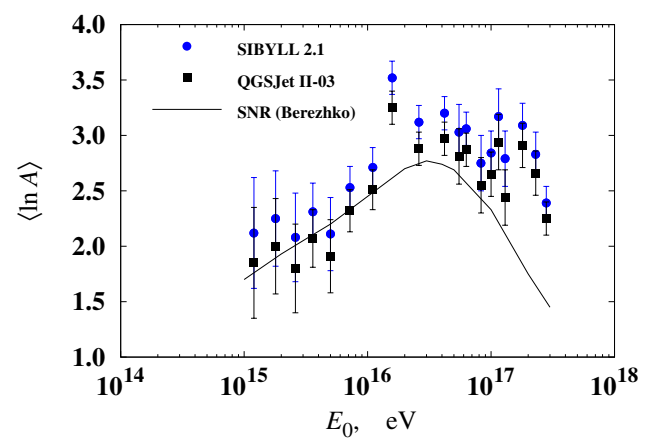

Figure 5. The average mass, $\langle\ln A\rangle$, as a function of energy. Models SIBYLL 2.1 and QGSJET II-03 are used to estimate $\langle\ln A\rangle$ basing on $X_{\max }(E)$ measured with the Yakutsk array.

\section{MODERNIZATION OF THE ARRAY}

The modernization program of the array is aimed at a set of items:

- Is the knee in energy spectrum due to the energy/mass distribution of GCR sources, or to the $\mathrm{CR}$ diffusion in $\mathrm{G}$ magnetic fields?

- What is the actual maximum energy of supernova remnant accelerators? 

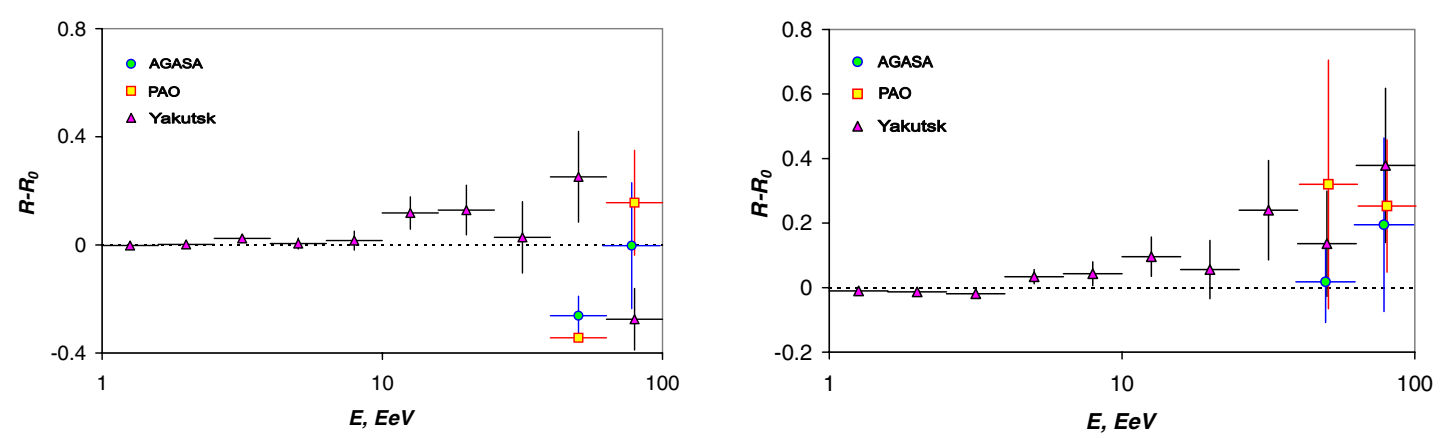

Figure 6. A search for excess flux from galactic (left panel) and supergalactic (right panel) planes.
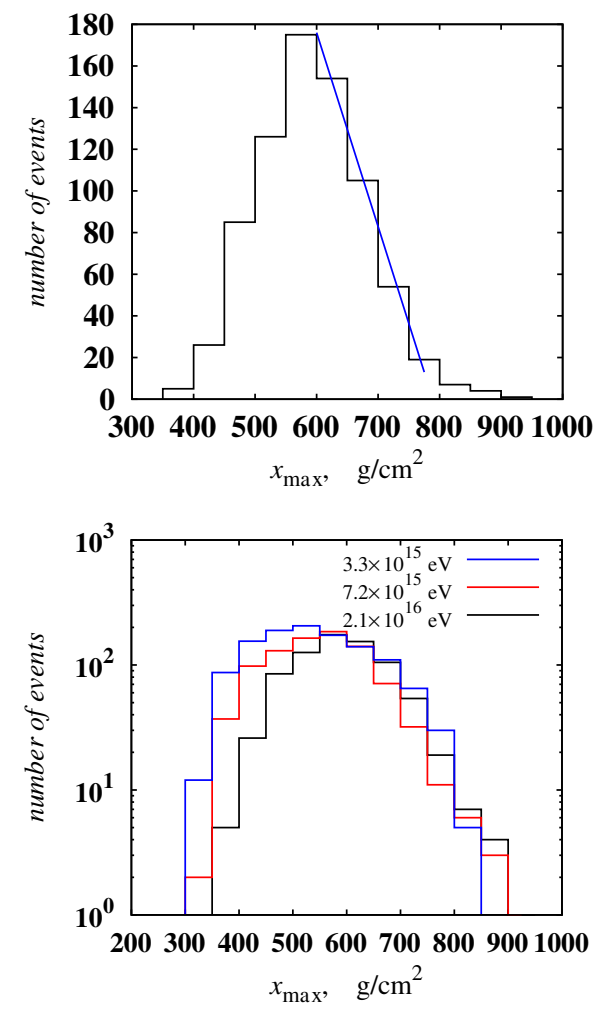

Figure 7. Upper panel: estimation of $X_{\max }$ distribution slope. Bottom panel: experimental $X_{\max }$ distributions.

- Where is the transition region between G \& EG components of CRs?

- Which features of CRs should be measured in a future to constrain a variety of CR acceleration and propagation models?

The key to this program is an accurate determination of the mass composition of CRs (Fig. 10), which is a weak point of existing EAS arrays. We plan to adapt the well-known imaging Cherenkov telescope technique to measure the angular and temporal structure of the signal connected to EAS longitudinal profile, and hence to $X_{\max }$ and the mass composition of CRs above $E=10^{15} \mathrm{eV}$ [16].

To accomplish these tasks we are going to make improvements to the array detectors and data acquisition system: LAN channel capacity increased to $1 \mathrm{Gbps}$; employ new scintillation detectors; 
EPJ Web of Conferences

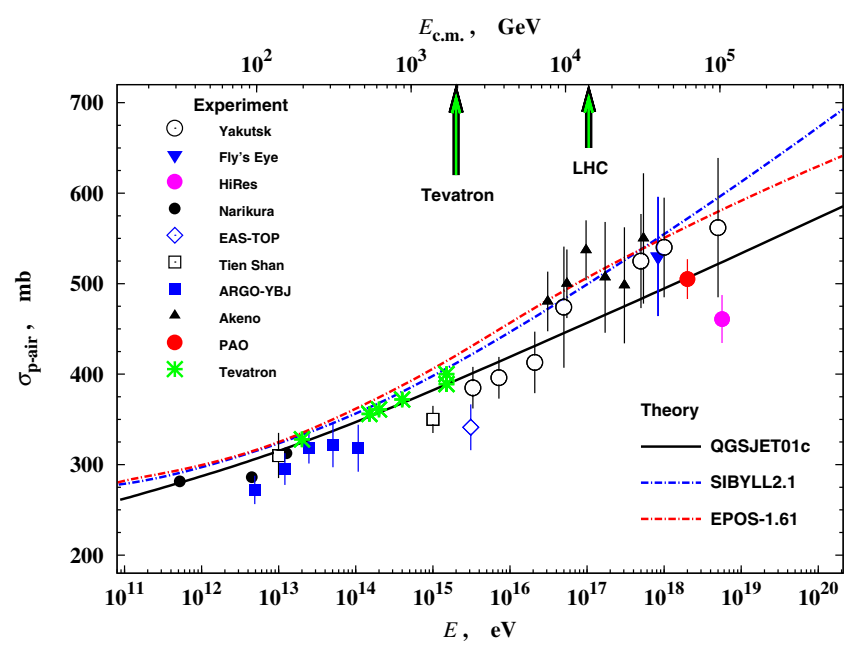

Figure 8. Total inelastic proton-air cross section. Experimental data and hadronic interaction model calculations.

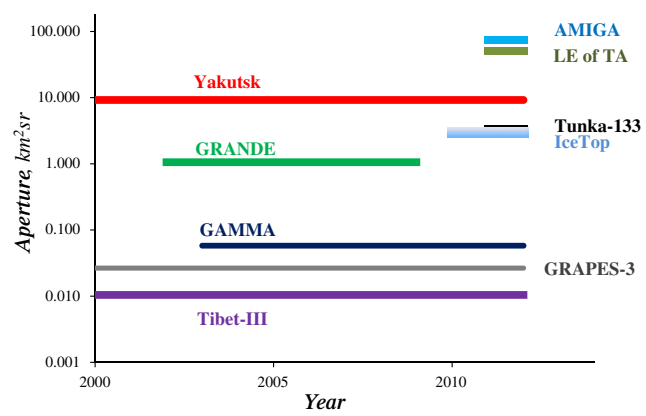

Figure 9. Comparing array apertures in the energy range $10^{14}-10^{17} \mathrm{eV}$.

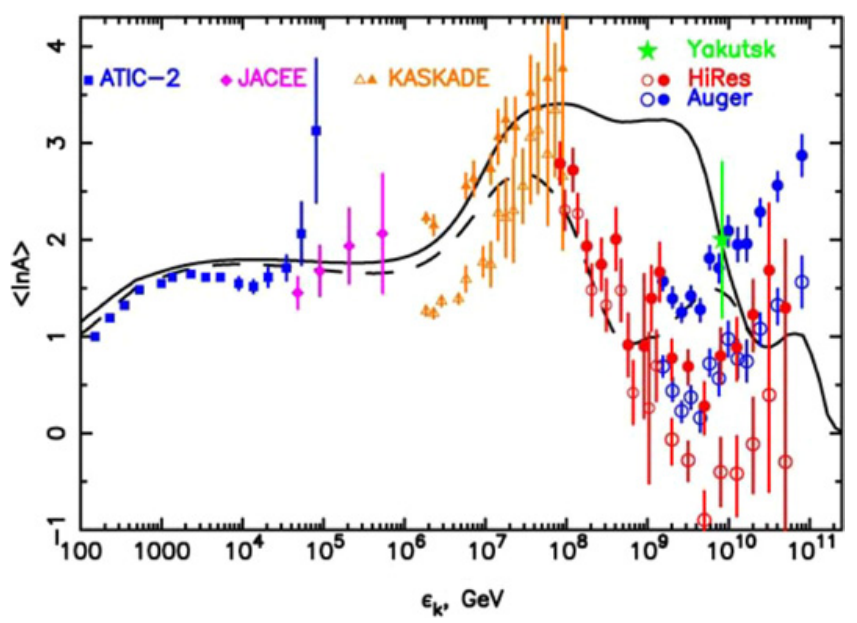

Figure 10. A set of experimental data on average CR mass vs ankle (solid curve) and dip (dash curve) scenario of CR origin (from [15]). 


\section{UHECR 2012}

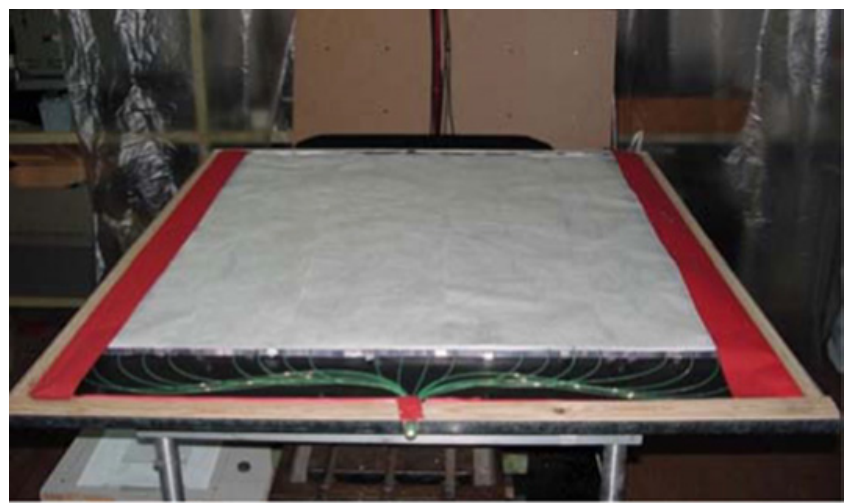

Figure 11. The detector made of plastic scintillator.

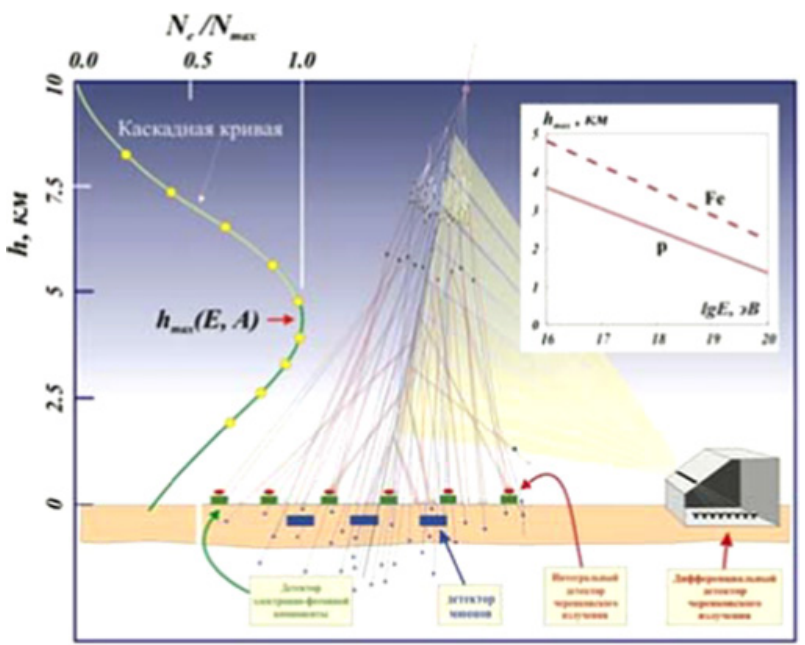

Figure 12. A scheme of camera obscura detector working.

assemble new station controllers to achieve the accuracy of timing $\sim 10 \mathrm{~ns}$; construct new differential detectors to measure the air Cherenkov light; modernize muon detectors; create a radio detection system of EAS in the frequency range from $\sim 10 \mathrm{GHz}$ to $\sim 10 \mathrm{MHz}$.

Particularly, soft component detector will consist of one layer $\left(1 \mathrm{~m}^{2}\right.$ area, $1-3 \mathrm{~cm}$ thickness $)$ plastic scintillator manufactured in the Institute of High Energy Physics, Protvino (Fig. 11). New stations in a triangular grid with appropriate spacing will consist of two detectors in metal housing. All the data will be transmitted to the central processor where the shower events have to be triggered via the signal coincidences.

A snapshot of the longitudinal profile of the cascade in Cherenkov light will be taken with the camera obscura detectors (Fig. 12) and wide FOV Cherenkov telescopes (Fig. 13). The angular and temporal structure of the signal will be used to locate $X_{\max }$ and to estimate the mass composition of CRs at energies above $10^{15} \mathrm{eV}[16]$.

To verify the ability of air Cherenkov light differential detectors to discriminate angular and temporal profiles of signals from showers initiated by primary nuclei and photons at energies above $10^{15} \mathrm{eV}$, we modeled the process. In Figs. 14, 15 the angular and temporal distributions of Cherenkov light in the detector at $R=800 \mathrm{~m}$ from the shower axis are shown. The EAS primaries are $P, F e$, and $\gamma$ with 


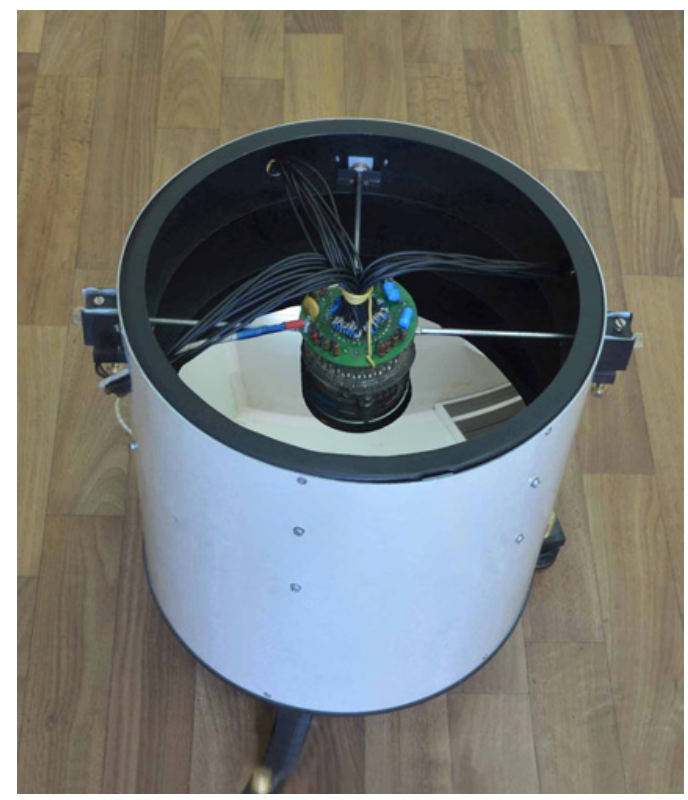

Figure 13. Wide FOV Cherenkov telescope.

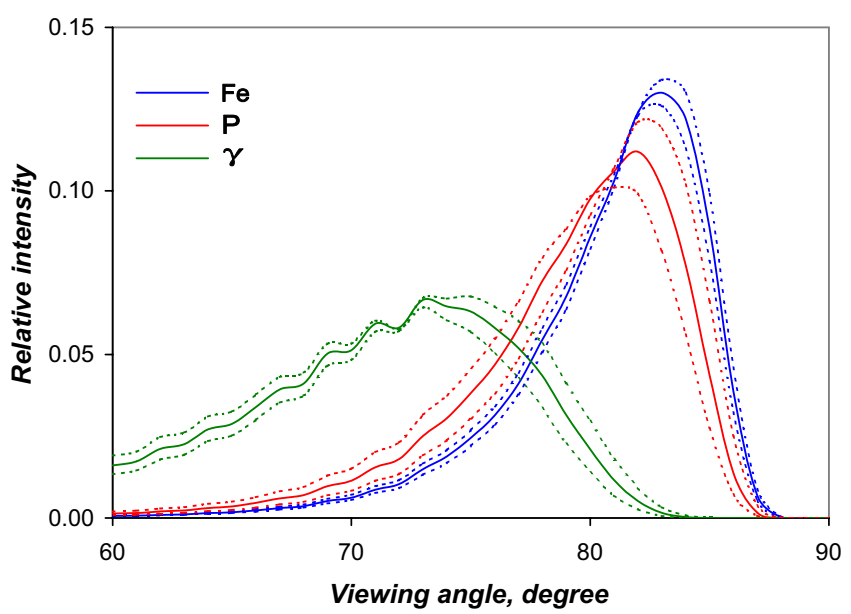

Figure 14. Angular profile of the Cherenkov signal from EAS.

$E=10^{15} \mathrm{eV}$. It appears from the Figure that the angular resolution $\sim 1^{0}$ and time resolution $\delta t<10 \mathrm{~ns}$ of the Cherenkov telescope are sufficient to distinguish the primaries, at least the $\gamma$-quanta from the nuclei, basing on the angular and temporal distributions of the signal.

The result of ray tracing within the telescope is shown in Fig. 16. The light intensity from a distant point source with slant angle $\alpha=7^{0}$ is shown as a function of coordinates on the camera surface. Pixel size is $3.75 \times 3.75 \mathrm{~mm}^{2}$. This demonstrates the ability of our prototype Cherenkov telescope to convert the angular distribution of light sources to the spatial distribution of signals on the imaging camera surface with adequate accuracy. 


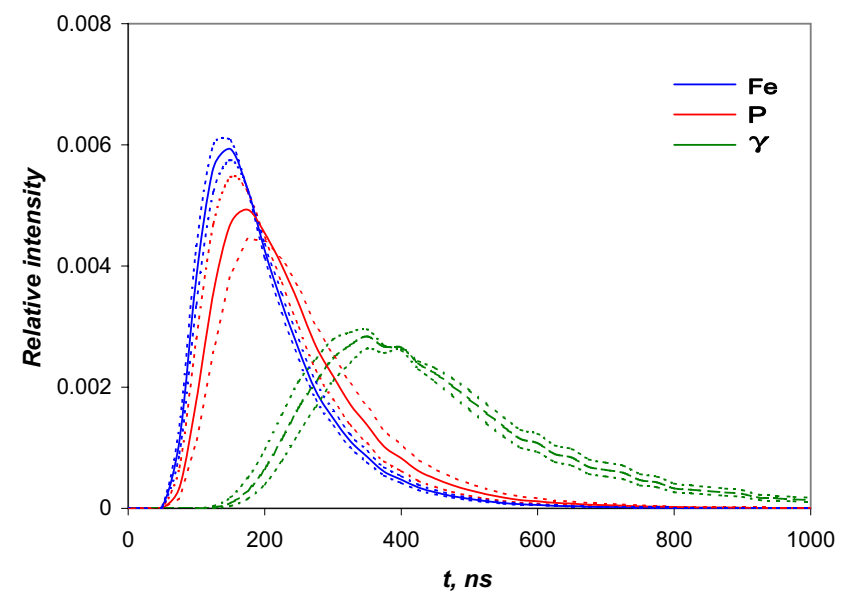

Figure 15. Temporal profile of the Cherenkov signal from EAS.

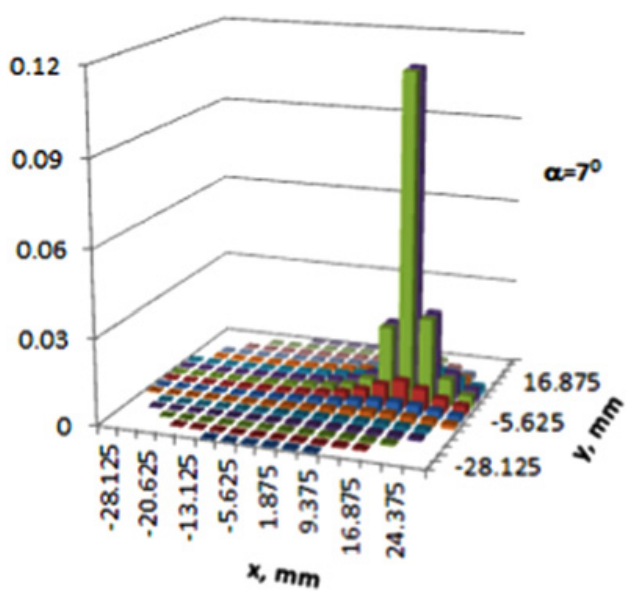

Figure 16. Image of a distant point source in the Cherenkov telescope.

\section{CONCLUSIONS}

- The main object to study with the Yakutsk array modernized is the transition region between $\mathrm{G}$ and EG components of CRs;

- Another task is to measure the intrinsic features of G component of CRs: energy spectrum and mass composition of GCRs will be measured with the Yakutsk array modernized. Sources of GCRs will be searched for;

- Longitudinal profile of the showers will be measured with differential detectors of air Cherenkov light from EAS;

- Another future direction is a co-operation with the CR community in dealing with a nextgeneration ground based detector of EG CRs.

The work is supported in part by SB RAS (integral project "Modernization of the Yakutsk array"), RFBR (grants 11-02-00158, 11-02-12193, 12-02-10005) and Russian Ministry of Education and Science (contract nos. 02.740.11.0248, 16.518.11.7075). 
EPJ Web of Conferences

\section{References}

[1] M.N. Dyakonov et al., Cosmic Rays of Extremely High Energy (Nauka, Novosibirsk, 1991)

[2] V.P. Egorova et al., Nucl. Phys. B (Proc. Suppl.) 136 (2004) 3

[3] A.A. Ivanov, S.P. Knurenko I.Ye. Sleptsov, New Journ. of Phys. 11 (2009) 065008

[4] A.A. Ivanov et al., Proc. 26th ICRC, Beijing 1 (2011) 186

[5] M. Nagano and A.A. Watson, Rev. Mod. Phys. 72 (2000) 689

[6] A.A. Ivanov, Astrophys. Journ. 712 (2010) 746

[7] S.P. Knurenko and A.V. Sabourov, This Conference, ID:43, (2012)

[8] J.R. Horandel et al., J. Phys.: Conf. Ser. 47 (2006) 41

[9] E.G. Berezhko, H.J. Volk, Astrophys. Journ. 661 (2007) L175

[10] G. de Vaucouleurs, Astron. Journ. 58 (1953) 30

[11] A.A. Ivanov, Nucl. Phys. B (Proc. Suppl.) 190 (2009) 204

[12] R.W. Ellsworth et al., Phys. Rev. D 26 (1982) 336

[13] S.P. Knurenko and A.V. Sabourov, This Conference, ID:42, (2012)

[14] S.P. Knurenko et al., Proc. 26th ICRC, Salt Lake City 1 (1999) 372

[15] E.G. Berezhko, S.P. Knurenko and L.T. Ksenofontov, AstroPart. Phys. 36 (2012) 31

[16] A.A. Ivanov et al., Astroph. Space Sci. Trans. 6 (2010) 53 